\title{
Perceptions of the HPV Vaccine in Indian Immigrant Mothers
}

\author{
Ramya Chitturi ${ }^{1}$ and Kate Flowers Rossner ${ }^{1 \#}$ \\ ${ }^{1}$ Santa Clara High School, Santa Clara, CA, USA \\ \#Advisor
}

$\underline{\text { ABSTRACT }}$

Each year, over 14 million Americans are infected with human papillomavirus, or HPV. Yet, the 2020 US goal is to vaccinate $80 \%$ of all adolescents for HPV is over $25 \%$ higher than the actual vaccination rate of teens, which was $54.2 \%$ in 2019. In California specifically, Asians were found to have the lowest HPV vaccination rates compared to other ethnic groups. When looking at the major Asian subgroups, the Indian American population has grown by $76 \%$ between 2000 and 2010 in Santa Clara County. Yet, no previous studies have been conducted about the Indian immigrant population. Thus, this paper explores the following research question: How do Indian immigrant mothers in Santa Clara County, California perceive vaccinating their daughters for HPV? A phenomenological study using oneon-one virtual interviews was conducted using two question frameworks to structure the interview questions: the Socio-Context framework and Andersen's Behavioral Model of Health Services. Indian immigrant mothers' were found to significantly prioritize their daughter's safety. Another notable theme was that there was a strong trust in the overall science of vaccines to keep the daughters safe. Although many mothers believed that the HPV vaccine was a decision solely between the parents and the doctor, some mothers believed that their community would be influenced fairly strongly by the actions of their family and friends regarding vaccinating their children for HPV. Overall, this study examined the HPV vaccine perception in an understudied population, Indian immigrant mothers in California.

\section{Introduction}

Each year, over 14 million Americans are infected with human papillomavirus, or HPV. HPV is the most commonly sexually transmitted infection (STI) in America. An STI is defined as a preventable infection that can be passed from one person to another through intimate skin-to-skin contact. The CDC reports that over half of newly contracted STIs in America were developed among youth between the ages of 15 and 24. HPV has several different variants that can lead to various types of cancers in the most extreme cases. Although men can also contract HPV, 38.4\% of all American women aged 15 to 59 are infected with HPV (CDC). In terms of side effects, cervical cancer in women is the most prevalent cancer caused by HPV in women. Specifically, chronic HPV infection causes $99.7 \%$ of all invasive cervical cancers for women (Clifford et al. 2006).

In 2006, the HPV vaccination was licensed in the United States, and it was recommended that this vaccine should be administered to all children at ages 11 to 12 , ideally prior to any sexual activity in order to maximize its effectiveness (CDC). In the eight years after the vaccine was licensed, there were more than 67 million doses of the HPV vaccine administered in America (White, 2014). Out of those 67 million doses given, only about 25,000 of those cases ended in adverse reactions according to reports by the Vaccine Adverse Event Reporting System, which is part of the U.S. Department of Health and Human Services (White, 2014). Despite the prevalence of HPV and the general harmlessness of the vaccine, the most recent studies report that only $54.2 \%$ of all adolescents aged 13 to 17 in the United States were up to date on the HPV vaccine in 2019 (Elam-Evans et al., 2020). This statistic greatly differs from 
the target of vaccinating $80 \%$ of all American adolescents by 2020 set by Healthy People, an organization managed by the U.S. Department of Health and Human Services.

\section{HPV Vaccination Factors}

An important factor in determining whether children are vaccinated for HPV is parent involvement, especially since the HPV vaccine is first recommended to be administered when the child is a minor. It was found that women make the majority of healthcare decisions for their children, with $81 \%$ of mothers taking their children to doctor appointments compared to just 16\% of fathers (Ranji and Salganicoff, 2013). For HPV in particular, over $90 \%$ of mothers are involved when making the decision about vaccination, with about $40 \%$ of them being the sole decider in this process (Berenson et al., 2014), typically making mothers the primary decision makers for vaccinating their children for HPV.

In general, the HPV vaccination is most effective when given prior to the onset of sexual activity. According to the CDC, HPV infection rates decreased by $71 \%$ in women in their early 20 s and by $86 \%$ in female teens between the ages of 14 and 19 from the years of 2006 to 2016, the 10 years following the licensing of the HPV vaccine. Although many women can contract HPV thorugh sexual activity as early as their teens, parents do not think of their own children as sexual beings and therefore do not believe that their child is or will be having sex as an adolescent (Elliot, 2010). This may lead them to believe that their children are sexually active later than the CDC's calculated national average of approximately 17 years. If parents don't understand when their children become sexually active, they may be unable to successfully agree to administer the HPV vaccine prior to any sexual activity, leaving teens at higher risk for contracting HPV without the vaccination.

\section{HPV Vaccination Rates by Group}

In order to actually measure HPV vaccination rates by demographics such as ethnicity in more detail, several studies were conducted in America. The various ethnic demographics can be studied to see which ones have the lowest rates since and note the factors unique to that demographic. A statewide analysis of California's HPV vaccination rates found that Asians parents were only 60.5\% likely to vaccinate daughters before age 13 for HPV, compared to $74.1 \%$ of white parents and $84.1 \%$ of Hispanic parents (Constantine et al., 2007). The study's results demonstrate a difference in vaccination rates based on ethnicity, indicating that each ethnic group likely has different perceptions of the vaccine that contribute to their final decision about choosing or not choosing to vaccinate their daughter. However, this analysis did not account for the various subgroups of each ethnicity, thus possibly overlooking key differences in why certain groups had lower vaccination rates than others. Subgroups refer to variation within a broader ethnic group due to a difference in sociodemographic factors, such as nationality, language, or religion (Birnbaum-Weitzman, 2013).

Within the broad Asian ethnic group indicated to have the lowest HPV vaccination rate in California, Pew Research Center indicates that there are 19 subgroups that account for $94 \%$ of the total Asian population in the United States. Although the Asian demographic was indicated to have the lowest vaccination rate for daughters under age 13, the variations between the various Asian subgroups' vaccination rates were overlooked. Therefore, the perceptions of the various ethnic subgroups that contribute to the Asian population and the cultural differences that contribute to their potentially varying perspectives on the HPV vaccine were not considered.

\section{Indian American Perceptions}

Among all Asian Americans, the Indian American population is a key subgroup that is rapidly growing. In California, Indians are the fourth most populous Asian subgroup. According to the U.S. Census Bureau, the IndianAmerican population in Santa Clara County specifically has grown by $76.1 \%$ between 2000 and 2010, making them a crucial ethnic group when considering HPV vaccination rates among the overall Asian population. 
Despite this growth in population, no studies have focused on the Indian population in California specifically, contributing to a lack of understanding about the reasons Indian mothers choose or choose not to vaccinate their daughters for HPV. However, looking at similar studies about people who live in India, along with Asians and immigrants living in America, can give some preliminary insight into what the current perspectives of Indian immigrant mothers may be. In India, premarital sex is strongly discouraged while lifelong monogamous relationships are encouraged, especially among women (Subaiya, 2008). This would cause most parents to assume that their daughters will not be sexually active before marriage (Subaiya, 2008). This could cause many Indian parents to believe that their children will not need the vaccine since the probability that they would be exposed to HPV is much lower due to the minimal number of sexual partners.

Another study by Netfa et al. in 2020 conducted a systematic review of immigrant parents' perceptions of the HPV vaccine. A common trend in the studies covered by the review was that there was a very low understanding of the HPV vaccine and its purpose. This was further observed in a study that looked at the awareness of HPV in students and their parents in India, with only $15 \%$ of the students and parents who participated showing awareness of HPV and cervical cancer (Hussain et al., 2014). So there is a lack of HPV knowledge in India, but there is limited understanding about whether this is the case for Indian immigrants in America as well. These studies ultimately propose that a reason for the low vaccination within Indian immigrant families could potentially be the lack of knowledge and must be studied further. Yet the perceptions of immigrant parents were not completely negative, as further demonstrated in Netfa et al.'s systematic review. Parents were much more willing to accept the vaccine after it was recommended by their doctors or if they felt that the vaccine would be approved by their community, so these factors must also be considered within the Indian immigrant population specifically. Finally, studying the perceptions of mothers who have both chosen to vaccinate their daughters for HPV and have also not done so is important in order to understand the perceptions of the Indian immigrant mother population as a whole. Despite these potential reasons why HPV vaccination is so low in the Indian immigrant population in America, there have been no studies conducted that explicitly look at this population. Therefore, research must be conducted to see if these factors apply to this population as well, since they may be uniquely affected after immigrating to Santa Clara County.

Thus, this study investigates the following research question: How do Indian immigrant mothers in Santa Clara County, California perceive vaccinating their daughters for HPV? By understanding these perceptions, HPV vaccination rates in the Indian population in America can be improved by catering vaccine interventions with their needs in mind.

\section{Methods}

\section{Participants}

Purposive sampling was used to recruit 8 participants to participate in virtual open-ended interviews for this phenomenological study. Purposive sampling, which is a sampling method that results in a non-random sample of participants with certain common characteristics, was used to find people who met the following criteria:

- Mothers with a daughter from ages 11 to 18

- Born in India but currently live in Santa Clara County in California

- Have been living in the United States for at least eight years

Mothers were recruited by word-of-mouth through appropriate contacts of the researcher and recruitment emails at local middle and high schools. Two of eight participants reported to not have vaccinated their daughters for HPV, while the other six have vaccinated their daughters. None of the mothers reported that they have contracted HPV in the past or indicated that they knew anyone who did, demonstrating that there was no significant personal connection that impacted their view of the vaccination. 
Eight participants were determined to be sufficient for the study since each focus group in the base study by Ko et al. had about ten participants, each from a different East African country. Since this study only focused on women of one ethnic origin, about eight to ten participants were deemed appropriate. One-on-one interviews were determined to produce the most relevant information by helping the interviews feel more comfortable and personal, compared to online questionnaires or other methods for data collection. The majority of previous studies that evaluated mothers' perceptions of the HPV vaccine also used interviews to collect qualitative data.

\section{Procedure}

Interviews were carried out from January to February 2021 through Zoom to maintain COVID-19 social distancing protocols. Interviews lasted approximately 20 to 30 minutes and were digitally recorded on a password protected computer.

Before the interview, each participant filled out a voluntary consent form through Google Forms and signed up for an interview slot if they consented. The form clearly outlined the purpose and procedures of the study and the participant's role if they choose to participate, along with potential risks and benefits. It also included contact information of the researcher and confidentiality procedures being implemented in order to maintain interviewees' privacy and anonymity.

All interviews were initially transcribed by the Otter.ai software but closely revised by the researcher to fix transcription errors. Each participant was asked the same questions in the same order. They were also given some general information from the CDC about HPV and its vaccine towards the start of the interview. Each interview ended with a link to the researcher's school's wellness center with a list of resources in case interviewees were troubled by the questions in the interview and wanted to reach out for assistance.

\section{Question Development}

The first section of questions were created by the researcher. These questions were used to gain a general idea of the interviewee's knowledge of the HPV vaccine and where they got this information from. These questions were followed by general information about HPV and its vaccine in order to make sure the interviewees had correct and thorough information to answer more personal opinion questions. Next, questions were asked to elicit background information about the interviewees and their perceptions. This included vaccination status and/or plans to vaccinate their daughter, the average age they believed women would be sexually active by, attitudes towards other vaccines, and implications of taking the HPV vaccine for their daughter.

The second and third sections of the interview were based on questions that were adapted from another qualitative study on mothers' HPV vaccine perceptions by Ko et al. in 2019. This reference study also interviewed immigrant mothers to study their perceptions of the HPV vaccine. However, Ko et al.'s study was different since it studied the perceptions of East African immigrant mothers, and interviews were conducted in focus groups. In contrast, this study focuses on Indian immigrant mothers and interviewed participants individually. One-on-one interviews were chosen over focus groups because this study only considered women of one ethnic origin unlike the foundational study.

The second section of the interview was based on the Socio-Context Framework. The Socio-Context Framework uncovers contextual factors in social, cultural, and religious areas that influence individual beliefs and health behavior (Pasick et al. 2009). The third and final section was based on Anderson's Behavioral Model of Health Services. Andersen's model outlines predisposing characteristics, enabling resources, and need-for-care factors (Andersen, 1995). Predisposing characteristics include health beliefs and social structures. Enabling resources include personal and community resources. Need-for-care factors include the perceived need for medical care. The researcher determined that these two frameworks were determined to be an effective way to evaluate the mothers' attitudes 
towards the HPV vaccine based on the prior study by Ko et al. and found that it aligned with the overall factors that were predicted to contribute to the mothers' perceptions found in other studies. Some of the factors from these frameworks demonstrated to be more significant than others, as will be further discussed in the Results section.

Finally, the interview concluded with participants sharing any final beliefs about the HPV vaccine to give them a chance to discuss other factors that may not have been explicitly addressed by the questions. A complete list of interview questions is included in Appendix A. The entire list of questions developed for this study was sent to the researcher's expert advisor for feedback before the first interview. The expert advisor is a pediatrician at a local hospital and has had several years of experience with educating parents about the HPV vaccine.

\section{Coding Data}

The frameworks allowed the questions to be organized by specific factors, and themes were drawn within each factor by finding significant commonalities or differences in the participants' opinions. Broad themes were pre-coded based on the two frameworks based on the following categories of factors and were later refined given the specific data found: contextual, enabling, predisposing, and general perceptions. A Google Sheets document was set up so that each spreadsheet corresponded to a main theme. Subthemes were indicated on the rows of the sheet, and each column represented a participant. Quotes and other relevant data were provided on the corresponding spreadsheet cell. Interview transcripts were closely studied to unearth subthemes and find common attitudes or experiences between the participants.

\section{Results}

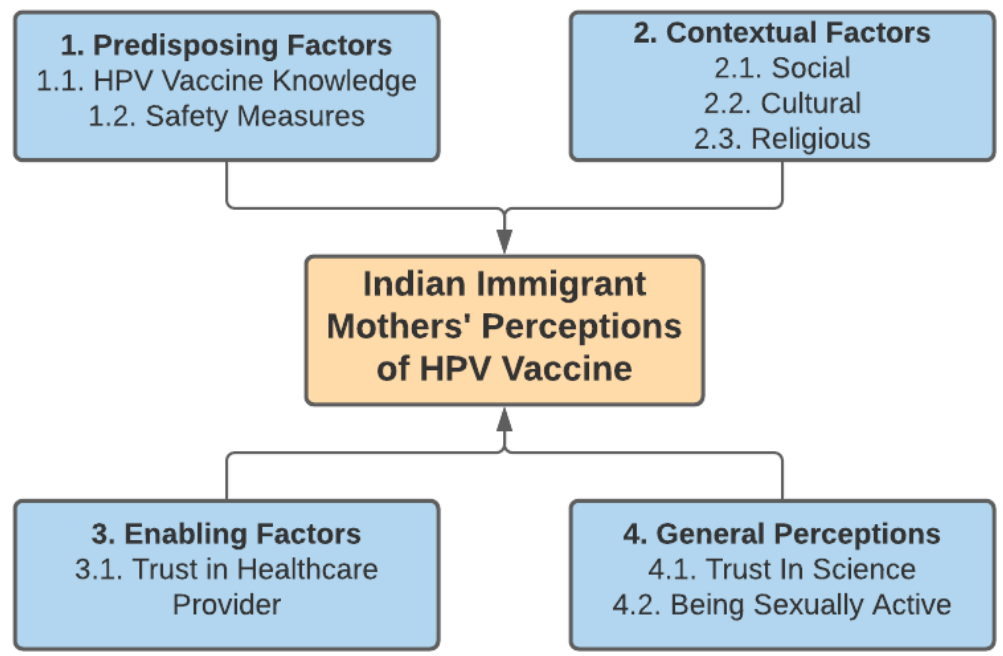

Figure 1. Themes and subthemes developed from the coding process.

Figure 1 outlines the findings that emerged after coding the eight interviews in the study. The four boxes on the outside indicate the main themes in bold (predisposing, contextual, enabling, and general perceptions) that ultimately contribute to Indian immigrant mothers' perceptions of the HPV vaccination. Subthemes for each of these factors are also listed in the box as bullet points. Themes are labeled based on the order that they are discussed in this paper for easier reference. To protect privacy, each participant's quotes are attributed to them using a number. 


\section{Predisposing Factors}

\subsection{HPV Vaccine Knowledge}

Overall, two of eight participants have not vaccinated their daughters for HPV yet, but they expressed support for the vaccine and indicated that they planned to do so soon.

In terms of HPV knowledge, some participants indicated that they did not completely understand the vaccine themselves; others responded that they believe others in the community do not understand the HPV vaccine fully either. For example, one participant was unaware of the recommended age to be vaccinated prior to the information provided during her interview and expressed some concern.

"Is there any particular reason that they started [at] 11 and 12? It's such a young age. I know for the girls the puberty might have started, but [it's] such a young age." (Participant 1)

There was also confusion among some participants regarding HPV and how it can be transmitted. A participant mistakenly believed that HPV can be transmitted through swimming in public pools and indicated it as a primary reason of giving her daughter the vaccine:

"I was so scared of swimming, because I heard so many things about the water and bacterial infection. So as soon as the doctor [mentioned the vaccine] I said, 'Okay, that's it." (Participant 3)

Despite this misinformation, six of eight participants agreed that HPV vaccination rates would increase if their community understood the vaccine better and the science surrounding vaccines in general. In addition, two participants attributed higher parental education levels or professions that required a higher level of education overall with a higher acceptance of the HPV vaccine:

"[People with only a high school education level's] view of things might be different from the ones that have [a] bachelor's degrees and above." (Participant 6)

\subsection{Safety Measures}

Most participants agreed that giving their daughter the HPV vaccine was not an implicit symbol that allows them to have sex. Rather, the mothers viewed it as a safety precaution in order to protect them in the future. A mother who vaccinated her daughter explained how this connection between the vaccine and giving permission to be sexually active didn't exist:

"I don't think it has anything to do with [having a] sexual life, but it's just safeguarding the future. So I don't think a vaccine should even determine that.” (Participant 8)

\section{Contextual Factors}

\subsection{Social}

Over half of the participants agreed that friends and/or family could play into choosing to vaccinate their daughters for HPV to a certain extent. However, the final decision is between the parents.

"I mean of course I'm open to people's experiences and what they have had. But... these decisions are something that just me and my husband take together... HPV is really very personal so it wouldn't be something that would be discussed at a family event." (Participant 2)

The other three participants believed that their community would be influenced fairly strongly by the actions of their family and friends regarding vaccinating their children for HPV. One also added that for parents that are hesitant about the HPV vaccine, positive comments or experiences of others would be significant in helping them choose to vaccinate their child: 
"They're probably in denial. So maybe in those kinds of instances, if they talk to people who are more accepting of it, they might come to terms with it or see it better." (Participant 6)

\subsection{Cultural}

A few mothers mentioned that Indian parents may be conservative, thus not accepting the fact that their daughters may be sexually active-either currently or in the near future):

"There are probably a lot of families that are extremely conservative and who wouldn't, who are probably not acknowledging or coming to terms that, you know, the child might be the one [who is sexually active]." (Participant 6)

However, five of the participants stated that their daughter's health is more important than culture. Despite the conservative nature of many Indian parents, several participants said that this would not pose a barrier when it comes to their daughter's health or believing in science. One participant also addressed westernization of her values, saying that she follows the environment around her in terms of vaccination.

"I should not say that I'm from this place, so I don't want to get vaccinated or whatever. No, I just follow wherever I'm living... Sometimes things have to change." (Participant 1)

\subsection{Religious}

All 8 participants agreed that religion had no influence on them when deciding to give their daughters the HPV vaccine, thus considering religion and science to be completely separate from each other's influence.

"I don't think religion should play a role [in vaccination], and definitely doesn't play a role for us." (Participant 7)

Another participant paralleled this experience by saying that most people in the Indian community are highly educated, leading them to separate religion when it comes to their health:

"Even if they are religious I think most of them come from highly educated backgrounds in the community in the US. So I don't think religion even plays any kind of role in this stuff." (Participant 6)

\section{Enabling Factors}

\subsection{Trust in Healthcare Provider}

All eight of the interviewees agreed that their healthcare provider or doctors were extremely important in order for them to make a decision to vaccinate their child and obtain proper information about the HPV vaccination. Even parents who chose not to talk to others about their vaccine decisions reported that their doctor played a key role in their decision making:

"We rely on the information that we get from a healthcare provider a lot to make a decision. So, timely and accurate information coming from a healthcare provider is of immense importance." (Participant 2)

In addition, three mothers said that they wanted their doctor to share both the benefits and possible risks of the HPV vaccination before they made their decision:

"There are always positive as well as negative sides to any vaccine. So... from a healthcare provider it helps to know what the side effects are not only in the short term, but in the long run." (Participant 2)

One participant also brought up her implicit trust in her daughter's doctor to provide accurate and comprehensive information:

"I feel [that the healthcare providers have major importance] because you blindly trust them, right? Whichever provider you're going with, you trust them... So their opinion, suggestions, or inputs make a huge role in our decision." (Participant 1) 


\section{General Perceptions}

\subsection{Trust in Science}

All eight interviewees also shared that they supported vaccines with a very long duration of immunity in general, saying that their views of the HPV vaccine were not different from these other vaccines. Mostly, participants agreed to vaccinate themselves and their children without too much resistance in general, choosing to follow the advice of their doctor:

"I trust the science so I just go ahead. I do read about it and research about that, and mostly I will go with what the doctors say since I trust the scientists." (Participant 5)

All eight interviewees also expressed their overall support of the HPV vaccine itself when asked, either through vaccinating their daughters or planning to do so soon and at the doctor's recommendation.

\subsection{Being Sexually Active}

Even among the mothers that have vaccinated their daughters for HPV, they did not view their daughters as old enough to start having sex:

"She took the vaccine [because] I have her to protect her. But... she's still in high school and she doesn't have that kind of thinking right now. And I will try to impose my value systems onto her, so I don't tell her to go for all those things." (Participant 4)

When asked about the age that they believe women will be sexually active, the average of answers in interviewees was 20.1 years compared to the American national average of 17.1 years. Only two participants estimated an age that was less than the average.

\section{Discussion}

This study examined how Indian immigrant mothers perceive vaccinating their daughter for human papillomavirus, or HPV. Their vaccine perceptions were shaped by their home country of India and further influenced after immigrating to the United States. Two of eight mothers had not vaccinated their daughters for HPV but expressed that they would be doing so soon. Overall, the eight study participants had a general trust in vaccines in and in the U.S. healthcare system. A critical finding was that mothers prioritized the health of their children over any other factor that they were asked about, including cultural or social factors. Therefore, the effectiveness of the vaccine and understanding the positive aspects of the vaccine's purpose are key for other Indian immigrant mothers to make a positive decision when choosing to vaccinate their children. Even when asked about cultural or religious factors, or asked about the age that the mothers believed women would be sexually active, participants agreed that any other influences were not as impactful when compared to making a decision that would benefit the daughter's health the most. Ultimately, mothers made their decisions with a primary focus on whatever would protect their daughter the most in the future. In addition, the strong trust that Indian immigrant mothers demonstrated in their healthcare providers to keep their daughter safe means that providing accurate and timely information about the benefits and possible risks of the HPV vaccine is extremely important in influencing decisions. This further corroborates past research (Greenfield et al., 2015) that a physician's recommendation is the most important factor in a parent's vaccine decision. Healthcare providers could emphasize how the HPV vaccine protects the daughters' health both in the short run and long run when recommending the vaccine to potentially increase vaccination rates in this population.

The results also showed that Indian mothers believe that positive input from other members of the community can help parents who are hesitant to vaccinate their child to be more likely to make a positive decision. A study from the Netherlands similarly found that parents are more likely to accept the vaccine if their friends and family also accepted it (Bults et al., 2011). Therefore, healthcare providers can view mothers as a way to increase overall vaccine 
uptake by helping them develop a positive view of the vaccine that they can then share with their friends or family. This way, hesitant parents can be reached not only through their doctors but also through friends and family.

Religion and culture were found to be two separate contextual factors that contributed to perceptions. Although some participants expressed that religion played no role in their perceptions, culture did play a role. Despite Indian culture being historically influenced heavily by religion (Stafford, 2006), the two factors have differing impacts when it comes to health and vaccination. As mentioned previously, this may have been the case due to daughters' health taking priority over any other contextual factors.

\section{Limitations}

In this study, one limitation was that the need for care factors from Andersen's Model of Behavioral Services were difficult to develop. These factors can be categorized as the perceptions of disease risk or status. This was likely because women's perceptions of the vaccine and the severity of HPV were significantly dependent on the information provided by the healthcare provider. This frequently overlapped with enabling factors from Andersen's model and made it difficult to distinguish between the two.

One other limitation of this study was being unable to interview participants in person due to COVID-19. Inperson interviews were shown to be marginally better than virtual interviews in terms of number of statements produced (Sullivan, 2012). This may have led to the study's results not being as comprehensive as if in-person interviews were conducted. Given more time, more participants could also have been recruited to the interview to provide an even more diverse view of the perceptions of the HPV vaccination. However, the themes found in the eight interviews have reached noticeable saturation, meaning that further data collection is not absolutely necessary (Saunders, 2018).

\section{Future Research}

In the future, other contextual factors that contribute to Indian immigrant mothers' perception can be further studied in order to gain an even greater understanding of their perceptions. Research could be conducted into how much living in America impacted their perceptions compared to their beliefs before living in the U.S., although most of this study's participants indicated that they had no or very little of HPV and its vaccine before immigrating to America.

Also, three of this study's participants mentioned that they were liberal-leaning or considered themselves to be more liberal than the overall Indian community, and they expressed support of the HPV vaccine. According to the Public Policy Institute of California, Santa Clara County is regarded as one of the most liberal counties in California in 2020. This corroborates a study that has found that more liberal people are likely to trust their healthcare provider more, leading to a higher intent to vaccinate (Baumgaertner et al, 2018). Indian immigrant mothers' perspectives on the HPV vaccine can also vary in other parts of the United States that have different social values or are typically more conservative than Santa Clara County, so further studies would need to be conducted to understand the difference.

In addition, a 1996 study by Moore et al. predicted that socioeconomic status could be an extremely significant factor of healthcare decision-making. So for future research, socioeconomic status could be addressed more in the interview questions along with targeting mothers of different political or cultural ideologies.

Overall, this was the first study that delved into Indian immigrant mothers' perceptions in California, which allowed the study to capture the unique perceptions and experiences of the population. With this new understanding, doctors and other healthcare providers can focus their information and interventions by catering to the hesitations and priorities of this population, thus increasing HPV vaccination rates in Indian immigrant mothers. 


\section{Acknowledgements}

I would like to thank my AP Research teacher, Ms. Kate Flowers Rossner, for all of her support to help me refine my research. I would also like to thank Dr. Manisha Panchal for serving as my expert advisor and providing valuable feedback on the current background of my research from a healthcare provider's perspective.

\section{References}

Andersen, R. M. (1995). Revisiting the behavioral model and access to medical care: does it matter?. Journal of health and social behavior, 1-10. https://pubmed.ncbi.nlm.nih.gov/7738325/.

Baumgaertner, B., Carlisle, J. E., \& Justwan, F. (2018). The influence of political ideology and trust on willingness to vaccinate. PloS one, 13(1), e0191728. https://doi.org/10.1371/journal.pone.0191728.

Berenson, A. B., Laz, T. H., Hirth, J. M., McGrath, C. J., \& Rahman, M. (2014). Effect of the decision-making process in the family on HPV vaccination rates among adolescents 9-17 years of age. Human vaccines \& immunotherapeutics, 10(7), 1807-1811. https://doi.org/10.4161/hv.28779.

Birnbaum-Weitzman O. (2013) Minority Subgroups. In: Gellman M.D., Turner J.R. (eds) Encyclopedia of Behavioral Medicine. Springer, New York, NY. https://doi.org/10.1007/978-1-4419-1005-9 1437.

Cates, J. R., Brewer, N. T., Fazekas, K. I., Mitchell, C. E., \& Smith, J. S. (2009). Racial differences in HPV knowledge, HPV vaccine acceptability, and related beliefs among rural, southern women. The Journal of Rural Health, 25(1), 93-97. https://doi.org/10.1111/j.1748-0361.2009.00204.x.

Centers for Disease Control and Prevention. (2017, August 14). NSFG - Listing S - Key Statistics from the National Survey of Family Growth. Centers for Disease Control and Prevention. https://www.cdc.gov/nchs/nsfg/key statistics/s.htm\#vaginalsexual.

Centers for Disease Control and Prevention. (2020, March 17). HPV Vaccine Safety and Effectiveness. Centers for Disease Control and Prevention. https://www.cdc.gov/vaccines/vpd/hpv/hcp/safety-effectiveness.html.

Centers for Disease Control and Prevention. (2020, September 9). Safety Information for HPV Vaccine. Centers for Disease Control and Prevention. https://www.cdc.gov/vaccinesafety/vaccines/hpv-vaccine.html.

Clifford, G., Franceschi, S., Diaz, M., Muñoz, N., \& Villa, L. L. (2006). HPV type-distribution in women with and without cervical neoplastic diseases. Vaccine, 24, S26-S34. https://doi.org/10.1128/JCM.01981-07.

Constantine, N. A., \& Jerman, P. (2007). Acceptance of human papillomavirus vaccination among Californian parents of daughters: a representative statewide analysis. Journal of Adolescent Health, 40(2), 108-115. https://doi.org/10.1016/j.jadohealth.2006.10.007.

Elam-Evans, L. D., Yankey, D., Singleton, J. A., Sterrett, N., Markowitz, L. E., Williams, C. L., ... \& Stokley, S. (2020). National, regional, state, and selected local area vaccination coverage among adolescents aged 13-17 years-United States, 2019. Morbidity and Mortality Weekly Report, 69(33), 1109. http://doi.org/10.15585/mmwr.mm6933a1. 
Elliott, S. (2010). Parents' constructions of teen sexuality: Sex panics, contradictory discourses, and social inequality. Symbolic Interaction, 33(2), 191-212. https://doi.org/10.1525/si.2010.33.2.191.

Greenfield, L. S., Page, L. C., Kay, M., Li-Vollmer, M., Breuner, C. C., \& Duchin, J. S. (2015). Strategies for increasing adolescent immunizations in diverse ethnic communities. Journal of Adolescent Health, 56(5), S47-S53. https://doi.org/10.1016/j.jadohealth.2014.10.274.

Hussain, S., Nasare, V., Kumari, M., Sharma, S., Khan, M. A., Das, B. C., \& Bharadwaj, M. (2014). Perception of human papillomavirus infection, cervical cancer and HPV vaccination in North Indian population. PLoS One, 9(11), e112861. https://doi.org/10.1371/journal.pone.0112861.

Jeudin, P., Liveright, E., Del Carmen, M. G., \& Perkins, R. B. (2013). Race, ethnicity and income as factors for HPV vaccine acceptance and use. Human vaccines \& immunotherapeutics, 9(7), 1413-1420.

https://doi.org/10.4161/hv.24422.

McCaffery, K., Forrest, S., Waller, J., Desai, M., Szarewski, A., \& Wardle, J. (2003). Attitudes towards HPV testing: a qualitative study of beliefs among Indian, Pakistani, African-Caribbean and white British women in the UK. British journal of cancer, 88(1), 42-46. https://doi.org/10.1038/sj.bjc.6600686.

McGhee, E. (2021, January 26). California's Political Geography 2020. Public Policy Institute of California. https://www.ppic.org/publication/californias-political-geography/.

Netfa, F., Tashani, M., Booy, R., King, C., Rashid, H., \& Skinner, S. R. (2020). Knowledge, Attitudes and Perceptions of Immigrant Parents Towards Human Papillomavirus (HPV) Vaccination: A Systematic Review. Tropical Medicine and Infectious Disease, 5(2), 58. https://doi.org/10.3390/tropicalmed5020058.

Pasick, R. J., Burke, N. J., Barker, J. C., Joseph, G., Bird, J. A., Otero-Sabogal, R., ... \& Guerra, C. (2009). Behavioral theory in a diverse society: Like a compass on Mars. Health Education \& Behavior, 36(5_suppl), 11S-35S. https://doi.org/10.1177/1090198109338917.

Pew Research Center. Asian Americans. Pew Research Center. https://www.pewresearch.org/topics/asian-americans/.

Ranji, U., \& Salganicoff, A. (2014). Balancing on shaking ground: women, work and family health. California: The Henry J Kaiser Family Foundation. https://files.kff.org/attachment/balancing-on-shaky-ground-women-work-andfamily-health-data-note.

Saunders, B., Sim, J., Kingstone, T., Baker, S., Waterfield, J., Bartlam, B., ... \& Jinks, C. (2018). Saturation in qualitative research: exploring its conceptualization and operationalization. Quality \& quantity, 52(4), 1893-1907. https://doi.org/10.1007/s11135-017-0574-8.

Stafford, N. (2006). Finding Lost: The Unofficial Guide. Ecw Press.

Subaiya, L. (2008). Premarital sex in India: Issues of class and gender. Economic and Political Weekly, 54-59. https://doi.org/10.2307/40278237. 
Sullivan, J. R. (2012). Skype: An appropriate method of data collection for qualitative interviews?. The Hilltop Review, 6(1), 10. https://scholarworks.wmich.edu/hilltopreview/vol6/iss1/10.

U.S. Department of Health and Human Services. (2020, October 8). Immunization and Infectious Diseases. Immunization and Infectious Diseases | Healthy People 2020. https://www.healthypeople.gov/2020/topics-objectives/topic/immunization-and-infectious-diseases/objectives.

White, M. D. (2014). Pros, cons, and ethics of HPV vaccine in teens-Why such controversy?. Translational andrology and urology, 3(4), 429. https://doi.org/10.3978/j.issn.2223-4683.2014.11.02. 